\title{
An EM Modeling for Rescue System Design of Buried People
}

\author{
A. De Leo, ${ }^{1}$ V. Petrini, ${ }^{1}$ P. Russo, ${ }^{1}$ L. Scalise, ${ }^{2}$ V. Di Mattia, ${ }^{1}$ \\ Valter Mariani Primiani, ${ }^{1}$ and G. Cerri ${ }^{1}$ \\ ${ }^{1}$ Dipartimento di Ingegneria dell'Informazione (DII), Università Politecnica delle Marche, Via Brecce Bianche, 60131 Ancona, Italy \\ ${ }^{2}$ Dipartimento di Ingegneria Industriale e Scienze Matematiche (DIISM), Università Politecnica delle Marche, \\ Via Brecce Bianche, 60131 Ancona, Italy
}

Correspondence should be addressed to A. De Leo; a.deleo@univpm.it

Received 22 October 2014; Accepted 23 December 2014

Academic Editor: Diego Caratelli

Copyright (C) 2015 A. De Leo et al. This is an open access article distributed under the Creative Commons Attribution License, which permits unrestricted use, distribution, and reproduction in any medium, provided the original work is properly cited.

\begin{abstract}
The development of a rescue system for buried persons is a subject of growing importance in case of occurrence of natural disasters such as earthquake, landslides, or avalanches. In this paper a fully analytical model has been developed to get some fundamental a priori design characteristics. The proposed system is based on the detection of the victim movements due to its respiratory activity: in particular, when an electromagnetic (EM) wave impinges on a human body, the analysis of the reflected wave parameters such as amplitude, frequency, phase, or delay time allows for the detection of the breathing frequency. The model is simple on purpose because the great uncertainty concerning the characterization of many environmental parameters of a general situation makes a very detailed model useless. However, it is accurate enough to provide useful information about system design, filling the gap in the literature concerning the electromagnetic formulation of such kinds of problems. A system prototype was built using laboratory equipment to experimentally validate the model, and subsequently breathing frequency measurements were carried on, both in a lossless laboratory environment and in a lossy realistic scenario.
\end{abstract}

\section{Introduction}

Noncontact detection of vital signs is gaining a growing importance for its applications in many practical contests [1, 2], and in particular this work describes an electromagnetic system for the detection of buried human subjects by sensing their breathing activity. The working principle is based on probing the environment with electromagnetic waves and processing the echo signals: change of amplitude and phase of the reflected waves due to the fact that the respiratory activity allows for localizing the victim under debris or landslide.

Radio frequency (RF) systems for the detection of physiological activities can be grouped into two main families, Doppler radars and UWB systems.

Historically, Doppler systems for the detection of vital signs were first proposed. The idea consists in revealing thorax movements to obtain the respiratory frequency using radar techniques in the microwave region [3-6].

Most of the researches are related to the signal processing of the electromagnetic signal reflected by the human body and several techniques to enhance the extraction of vital signs were described and implemented $[7,8]$. More recent papers are essentially addressed to overcome existing difficulties concerning the applications to realistic environments, introducing effective processing strategies $[9,10]$, whereas other works deal with practical aspects related to the electronic device fabrication [11-13].

A different choice was offered by the capability of UWB radar pulses to pass through obstacles, due to their high resolution, low energy consumption, and immunity against multipath interference: these features were also exploited to extract human vital signs in complex environments [1416], including space scenarios $[17,18]$. Specific UWB radar systems [19-22] were designed for the rescue of victims trapped in rubble.

In this paper a new approach based on an electromagnetic point of view is proposed. In fact, in spite of many examples of RF systems for breathing and heart beat detection reported in the literature, the lack of an electromagnetic model, to describe the backscattering of a victim embedded in the surrounding environment, stands out. 


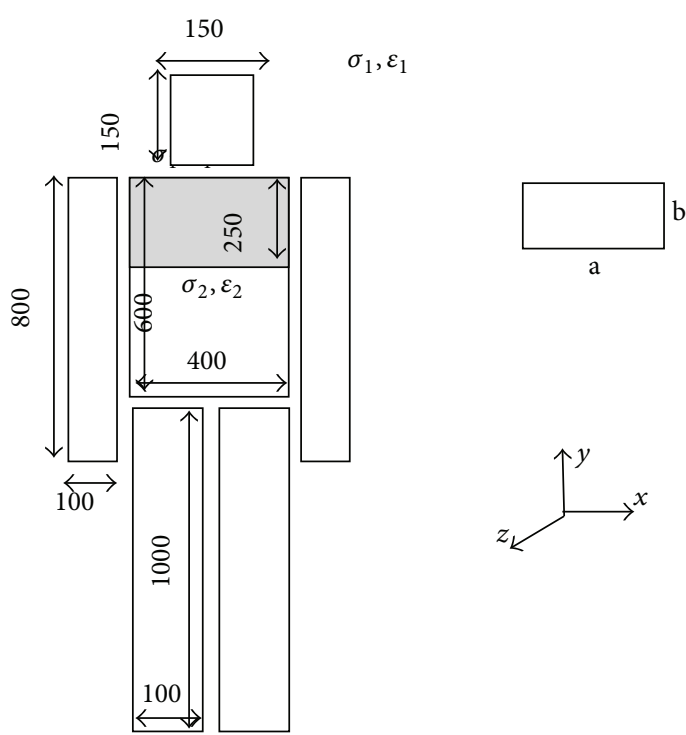

FIGURE 1: Scenario of the human body illuminated by an aperture antenna (dimensions are in $\mathrm{mm}$ ). The respiratory activity is simulated by a volume variation of 1 liter of the grey region.

The aim of the paper is to show that even a simple electromagnetic model is capable of providing useful information to address the system design, as operating conditions, the choice of the most suitable frequencies, and the sensitiveness.

In particular an analytical model for the scattering problem of a human body buried into a homogenous lossy medium will be presented. The approach is simplified, because a full wave solution of the electromagnetic problem does not seem to be necessary for our purposes and it would require a super computer to account for a detailed scenario. Moreover, a realistic environment is characterized by a great uncertainty related to geometrical and material parameters, so a more accurate modelling does not provide more accurate results. It must be remarked that we are mainly interested in the variations of the electromagnetic field due to the thorax respiratory movements rather than in the complete electromagnetic backscattering of the human body. However, this scenario is a good approximation of the situation of a man buried by a landslide or avalanche.

The model has been validated and the results compared with measurements both in a controlled environment and in a realistic scenario, as a road construction site.

\section{Electromagnetic Model}

The model is based on the evaluation of the current density induced by an electromagnetic wave impinging the human body. The knowledge of the body current allows for the computation of the backscattered signal.

For convenience the human body is replaced by a phantom with simplified human features (Figure 1). The different parts of the body are very close to each other but not electrically connected. The validity of these assumptions was demonstrated comparing the model prediction and the result of full wave numerical simulation [23]. In fact the current distribution inside all the parts of the human body is minimally affected by the electrical connections between two parts of the body, because in the operating frequency range the wavelength inside the human body is much smaller than the dimensions of the parts of the body. From a practical point of view this geometrical approximation allows us to treat each part separately providing a closed form solution for the electromagnetic problem.

The geometry of the problem and the reference system are shown in Figure 1. An aperture antenna of dimensions $(a \times b)$ radiates an electromagnetic field in a homogeneous medium 1 , having electric characteristics $\left(\sigma_{1}, \varepsilon_{1}\right)$. The wave impinges on a human body composed by $N_{P}=6$ homogeneous parallelepipeds, medium \#2, whose electric characteristics are $\left(\sigma_{2}, \varepsilon_{2}\right)$. The grey part of the thorax is supposed to vary its thickness ( $z$ dimension) of $\Delta z \pm 5 \mathrm{~mm}$ around its rest position to simulate a volume of 1 liter of air flowing during each respiratory act.

The electric field distribution $\overrightarrow{E_{\text {ap }}}$ on the aperture of the antenna is assumed to be uniform and polarized in the $y$ direction.

A good approximation [23] for the current density distribution $\overrightarrow{J_{\text {eq }}}$ to describe the thorax illumination due to an electromagnetic wave having the electric field $E_{\text {inc }} \hat{y}$ and propagating along the $\widehat{z}$ direction is

$$
\vec{J}_{\mathrm{eq}}^{(i)}=\left(\sigma_{2}-\sigma_{1}\right) \tau E_{\mathrm{inc}} e^{-((1+i) / \delta)\left(z-z_{1}^{(i)}\right)} \widehat{y},
$$

$z_{1}^{(i)}$ being the coordinate of the front surface of the $i$ th body part, $\tau$ the transmission coefficient at the interface between medium 1 and medium 2, approximated as an indefinite interface, and $\delta$ is the penetration depth of the field in medium 2.

The backscattered field can be easily calculated from the current density distribution $\overrightarrow{J_{\mathrm{eq}}}$ :

$$
\vec{E}_{\text {back }}=\eta \vec{H}_{\text {back }} \times \hat{r}=\eta\left(\frac{-j \beta \widehat{r} \times \vec{A}}{\mu}\right) \times \widehat{r},
$$

where $\beta$ is the wavenumber, using the far field approximation

$$
\vec{A}=\frac{\mu}{4 \pi} \sum_{i=1}^{N_{p}} \frac{e^{-i \beta r_{i}}}{r_{i}} \int_{x_{1}^{(i)}}^{x_{2}^{(i)}} \int_{y_{1}^{(i)}}^{y_{2}^{(i)}} \int_{z_{1}^{(i)}}^{z_{2}^{(i)}} \vec{J}_{\mathrm{eq}}^{(i)}\left(\vec{r}^{\prime}\right) e^{-i \beta \vec{r}^{\prime} \cdot \widehat{r}} d x^{\prime} d y^{\prime} d z^{\prime},
$$

where $\vec{r}^{\prime}$ is the position vector of a point of the infinitesimal element of the integration volume and $\vec{r}$ is the position vector of the observation point.

In (3) $x_{1}^{(i)}, y_{1}^{(i)}, z_{1}^{(i)}$ and $x_{2}^{(i)}, y_{2}^{(i)}, z_{2}^{(i)}$ are the Cartesian coordinates that define the $i$ th body element and $r_{i}$ is the distance between the center of the $i$ th body element and the antenna. It is worth mentioning that the choice of the geometry and the electromagnetic model was due to the possibility of achieving closed form expressions, nevertheless retaining a sufficient accuracy for the purpose of the paper.

The reflection coefficient $S_{11}$ measured at the antenna input port is the sought physical quantity because its time 
variations reveal the occurrence of a respiratory act of a buried subject.

Assuming the impedance matching between the generator and the transmission line, the reflection coefficient $S_{11}$ can be written as [24]

$$
S_{11}=\frac{V_{\mathrm{rec}}+V^{-}}{V^{+}}=\frac{\vec{E}_{\mathrm{back}} \cdot \vec{l}_{\mathrm{eff}}}{V_{g}} \frac{Z_{g}+Z_{0}}{Z_{0}+Z_{\mathrm{ant}}}+\Gamma_{\mathrm{ant}},
$$

where $V_{\text {rec }}$ is the received voltage at the antenna connector, $V^{-}$is the voltage reflected by the antenna and due to mismatching, $V^{+}$is the voltage incident at the antenna input port, $\vec{l}_{\text {eff }}$ and $\Gamma_{\text {ant }}$ are the effective length and the reflection coefficient of the antenna, respectively, $V_{g}$ and $Z_{g}$ are the generator voltage and impedance, and $Z_{0}$ is the characteristic impedance of the line connecting generator and antenna.

\section{Model Validation}

The aim of this section is to compare the levels of the backscattered signal measured and predicted using the analytical model. The intensity of the received signal as a function of the distance between the subject and the antenna provides a constraint in terms of system sensitivity.

The experimental setup consists of a double ridge horn antenna connected to a Vectorial Network Analyzer (VNA) located at a distance $D=3 \mathrm{~m}$ from the obstacle. The half power beamwidth of the actual antenna was used to set the aperture dimensions of the antenna used in the simulation. A Personal Computer (PC) acquired the VNA samples of the reflection coefficient and stored data for an offline processing. The obstacle, representing a human thorax, was a $400 \mathrm{~mm} \times$ $400 \mathrm{~mm} \times 50 \mathrm{~mm}$ Plexiglas box filled with salt water and placed at a distance of 2.5 meter from the antenna. To simulate the movement related with breathing activity the box position was changed of $1 \mathrm{~cm}$ in five steps of $2.5 \mathrm{~mm}$.

The VNA performed a frequency sweep from 1 to $9 \mathrm{GHz}$, because this is the maximum available range in our lab setup. The actual antenna impedance was measured at all the frequencies, and these values were used in the analytical model to determine the simulated reflection coefficient through (4).

In Figure 2 the comparison between the amplitude of the measured and the modeled $S_{11}$ is reported; in this case the comparison is made only at the distance of 2.5 meter because the amplitude variation of the reflected signal while shifting the obstacle of $1 \mathrm{~cm}$ is very small and all curves appear overlapped at a visual inspection. It can be observed that the model satisfactorily predicts the order of magnitude and the frequency dependence of the amplitude of the backscattered signal; random oscillations in the measured response are due to the multiple reflections of the environment and are not accounted by the model.

As regards the validation of the maximum phase variation prediction, the measurement procedure was carried out shifting the Plexiglas box of $1 \mathrm{~cm}$ (from $2.50 \mathrm{~m}$ to $2.51 \mathrm{~m}$ ) in order to simulate the movement related with breathing activity. Figure 3 shows the comparison between the measured and the calculated values for the maximum variation of the $S_{11}$ phase due to the target displacement. Comparing the two curves it

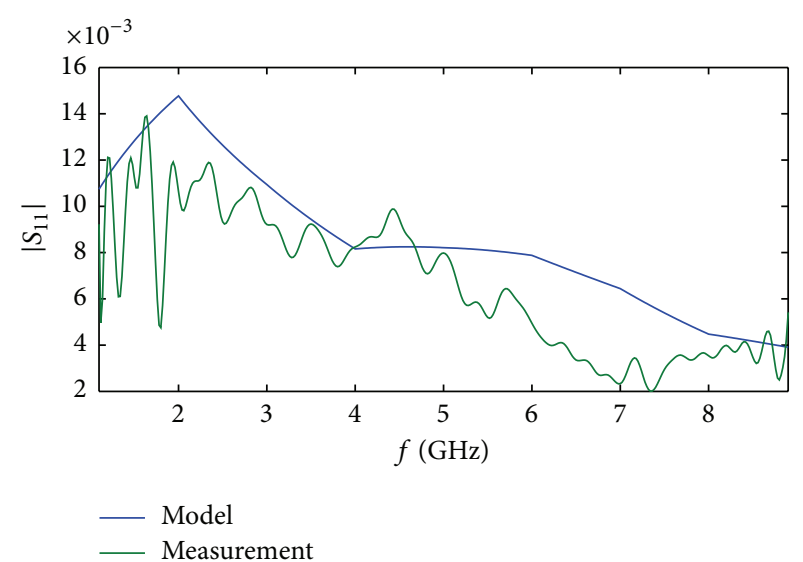

FIGURE 2: Comparison between the model prediction and the measurement for the module of the reflection coefficient.

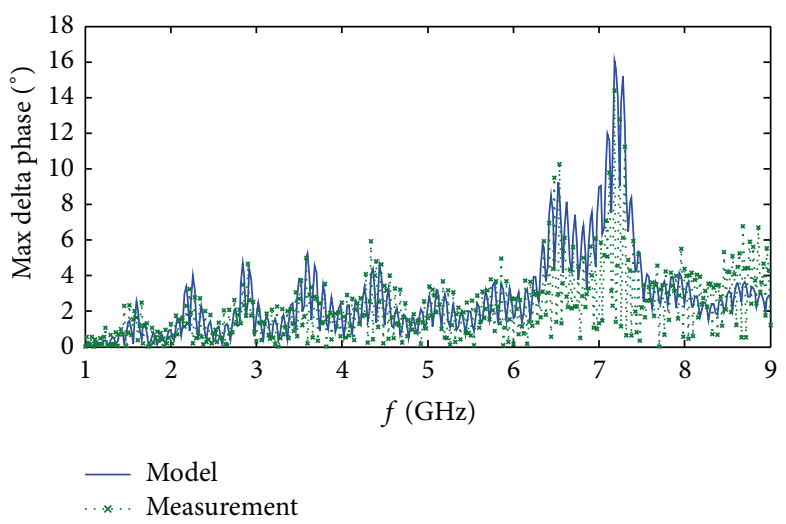

FIgURE 3: Comparison between the model prediction and the measurement for the maximum phase excursion of $S_{11}$ during a simulated respiratory act.

is possible to point out an appreciable agreement between the prediction of the model and the measured results.

As expected the higher the frequency is the greater the phase variation is with a maximum excursion of $16^{\circ}$ at about $7.3 \mathrm{GHz}$. However, it is worth noting that the choice of the working frequency seems to be critical, because maxima and minima of phase sensitivity are very close to each other.

\section{System Specifications}

The scenario of Figure 1 was implemented: the aperture antenna dimensions are $a=150 \mathrm{~mm}$ and $b=100 \mathrm{~mm}$ and it is placed at 3 meters of distance, facing the phantom thorax. The surrounding medium is a wet soil that according to [25] can be modeled as a homogenous dielectric having $3 \leq \varepsilon_{r 1} \leq$ 24 and $0.01 \leq \tan (\delta)=\varepsilon_{i 1} / \varepsilon_{r 1} \leq 0.05$, where $\varepsilon_{i 1}$ and $\varepsilon_{i r}$ are the imaginary part and the real part of the electric permittivity of the medium surrounding the victim. The human body is assumed to be a lossy dielectric with $\varepsilon_{r 2}=70$ and $\sigma_{2}=1 \mathrm{~S} / \mathrm{m}$. 


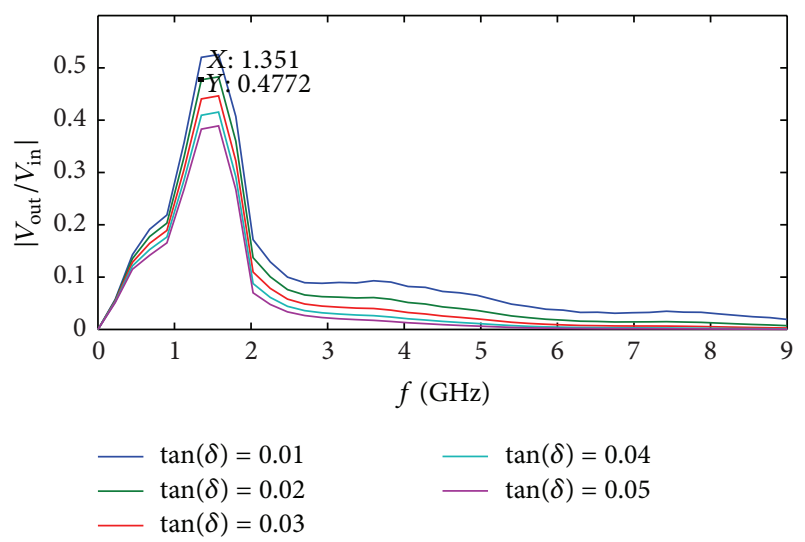

(a)

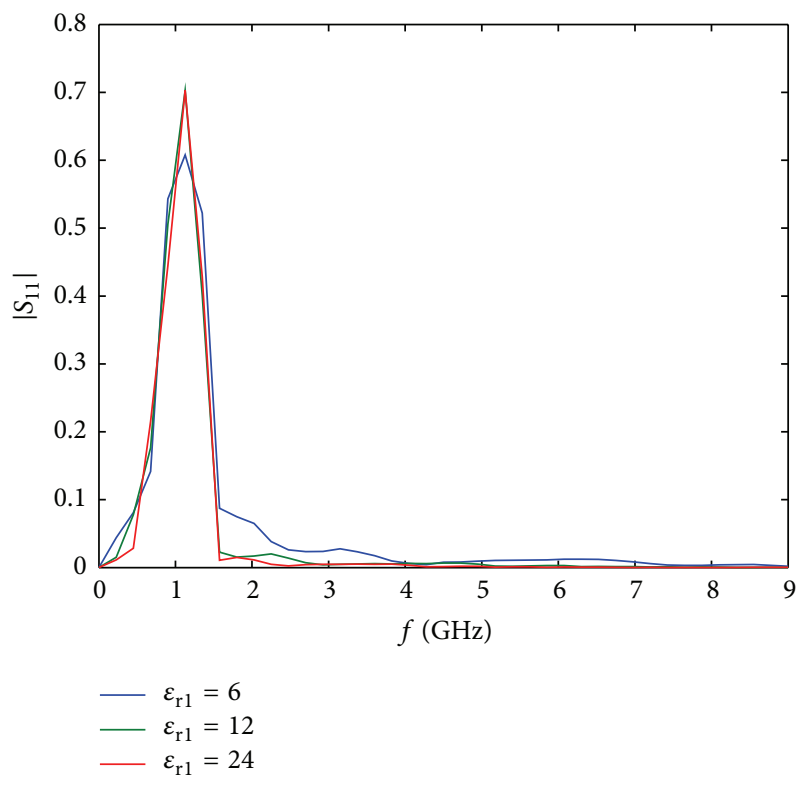

(b)

FIGURE 4: $\left|S_{11}\right|$ model prediction varying $\tan (\delta)$ (a) and $\varepsilon_{r 1}$ (b).

Figure 4(a) shows the $\left|S_{11}\right|$ for different values of $\tan (\delta)$, setting $\varepsilon_{r 1}=6$, whereas Figure 4(b) shows the $\left|S_{11}\right|$ for different values of $\varepsilon_{r 1}$, fixing $\tan (\delta)=0.01$. It can be observed that in both cases the frequency range $1-2 \mathrm{GHz}$ allows the system to have the maximum sensitiveness in amplitude. It is well evident that the soil attenuation at high frequencies and low antenna efficiency at low frequencies strongly dominate the $\left|S_{11}\right|$ behavior.

Summarizing the results predicted by the analytical model, it is possible to identify three different a priori system requirements: (a) an optimal frequency range from 1 to $2 \mathrm{GHz}$; (b) a frequency sweep analysis, more suitable than a continuous wave based system; (c) an antenna well matched with respect to the medium surrounding the buried victim.

Requirement (a) is related to the system maximum sensitivity (Figure 4). Requirement (b) is necessary because phase behavior is a rapidly oscillating function, and therefore

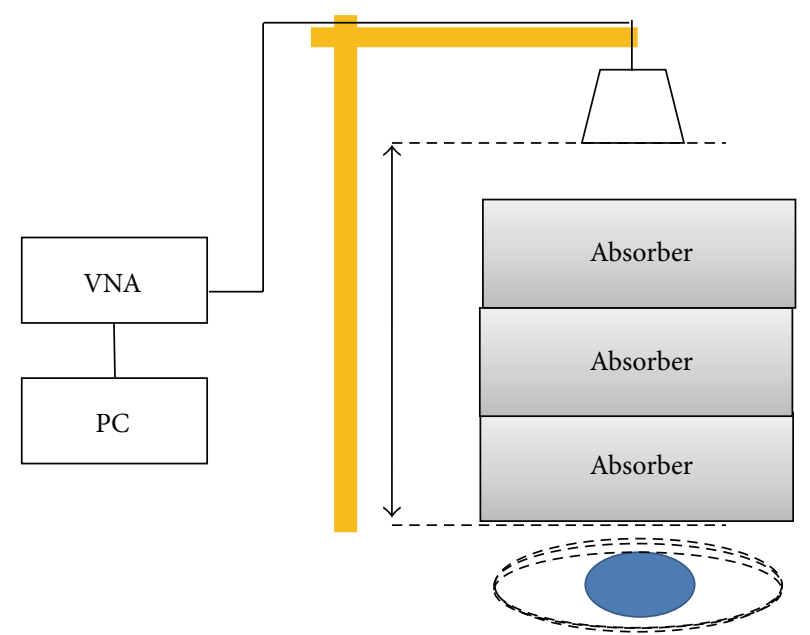

FigURE 5: Experimental setup for breathing measurement.

the system has to sample the subject at different frequencies to avoid the occurrence of blind frequencies (Figure 3). Requirement (c) is related to (4) that clearly shows how the antenna mismatching is a noise superimposed to the useful signal: obviously the matching condition has to be realized in working situation, with the antenna radiating the lossy medium.

\section{Breathing Detection}

Using the a priori system specifications predicted by the analytical model, a system prototype was built up with laboratory equipment to measure the breathing frequency of a human subject.

The measurements were carried out firstly in a controlled environment in our laboratory, in order to check the system functionalities, and subsequently in a realistic situation.

Concerning the laboratory measurements, whose set up is depicted in Figure 5, a person was placed on the floor and the antenna was hanged up by a dielectric structure at the height $D=2 \mathrm{~m}$. The antenna was placed so as to radiate an electric field parallel to the main dimension of the man. The first measurement was carried out in free space. Then, in each of the three next measurements, an absorbing panel was added, placing it above the victim, in order to simulate the losses of the medium that surrounds the victim. It was experimentally measured that the insertion of three absorbing panels introduces a total attenuation of about $20 \mathrm{~dB}$ corresponding to a depth of $5 \mathrm{~m}$ in case of dry soil [25].

The PC connected to the VNA stores the complex reflection coefficient for each frequency scanned, performing the measurements in $N_{f}=71$ frequency samples. Each scan lasts for $340 \mathrm{~ms}$ and during this time the subject can be considered still. A total number of $N_{s}=120$ scans are acquired for a measuring time of about $40 \mathrm{~s}$; in this way a matrix of values $\underline{\underline{s_{11}}}\left(f_{i}, t_{j}\right)$ is stored into the PC memory, where 


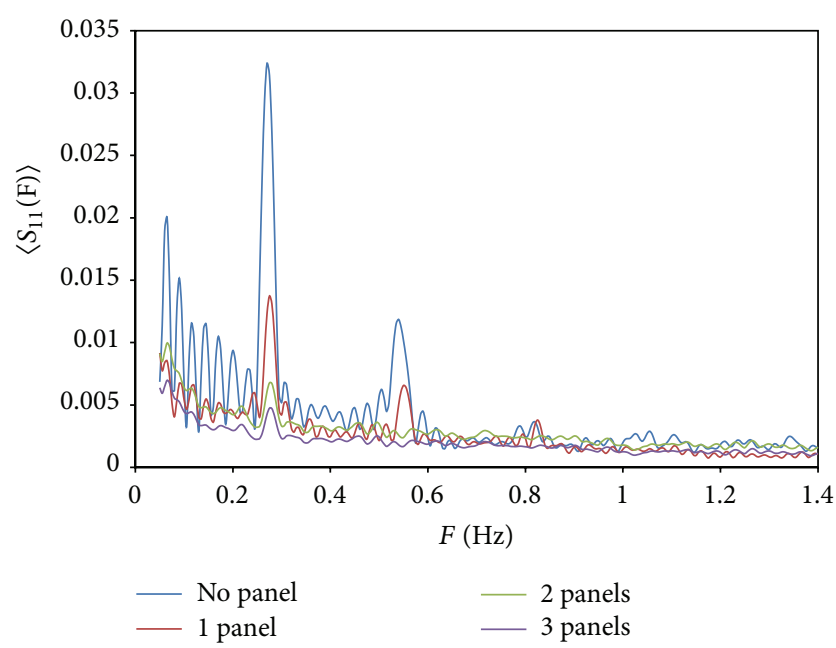

FIGURE 6: Measured breathing frequency.

$f_{i}\left(i=1, \ldots, N_{f}\right)$ is the $i$ th frequency sample and $t_{j}(j=$ $\left.1, \ldots, N_{s}\right)$ is the $j$ th acquisition time sample:

$$
\begin{aligned}
\underline{\underline{s_{11}}} & =\left[\begin{array}{cccc}
s_{11}\left(f_{1}, t_{1}\right) & s_{11}\left(f_{2}, t_{1}\right) & \cdots & s_{11}\left(f_{N_{f}}, t_{1}\right) \\
s_{11}\left(f_{2}, t_{1}\right) & \cdots & \cdots & \cdots \\
\cdots & \cdots & \cdots & \cdots \\
s_{11}\left(f_{N_{f}}, t_{1}\right) & \cdots & \cdots & s_{11}\left(f_{N_{f}}, t_{N_{t}}\right)
\end{array}\right] \\
& =\left[\begin{array}{llll}
\underline{s_{11}}(t) \underline{s_{11}^{f_{2}}}(t) & \cdots & \underline{s_{11}^{f_{n}}}(t)
\end{array}\right] .
\end{aligned}
$$

Each column of the matrix $\underline{\underline{s_{11}}}\left(f_{i}, t_{j}\right)$ represents the time variation of the complex reflection coefficient, due to breathing activity, measured at the frequency $f_{i}$. Performing a Fourier Transform (FT) of the $s_{11}$ values of each column, the $N_{f}$ frequency spectra $S_{11}(F)$ are obtained, where $F$ is a frequency in the breathing frequency range, typically $0.1-1 \mathrm{~Hz}$.

As the spectral content related to the breathing activity of each column is the same, an average value is evaluated to achieve a unique reconstructed signal: this simple technique allows us also to avoid the problem of the frequencies with very low sensitiveness.

Figure 6 reports the reconstructed spectra when none, one, two, or three absorbing panels are inserted. It can be observed that the sensed breathing frequency is about $F=$ $0.25 \mathrm{~Hz}$; this frequency can be detected also when all the three absorbing panels are inserted, even if the amplitude of the harmonics decreases as the losses increase. The lower part of the spectrum represents the effect of the very slow (and not related to the respiration) movements of the body during the 40 s measurement.

Concerning the breathing detection in a realistic scenario, a man buried by a landslide of excavated soil during the construction of a road was considered.

At first, we carried out the $s_{21}$ measurement to evaluate the path loss between points $\mathrm{A}, \mathrm{B}$, and $\mathrm{C}$ and the place where the victim was buried, inside a PVC pipe, Figure 7. In position A the antenna is at $2 \mathrm{~m}$ of distance from the victim, and in

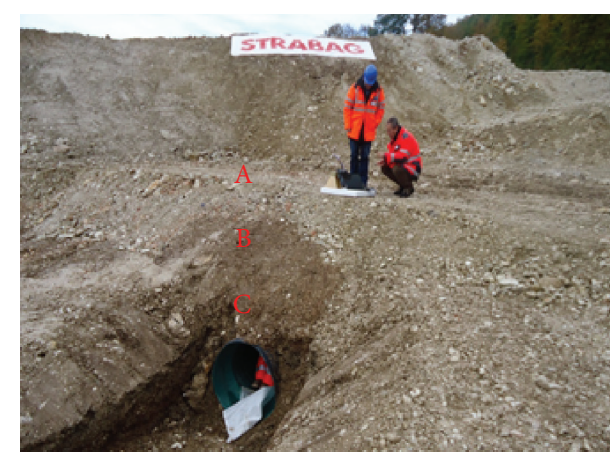

Figure 7: Test scenario: a victim is buried by $2 \mathrm{~m}$ of excavated ground.

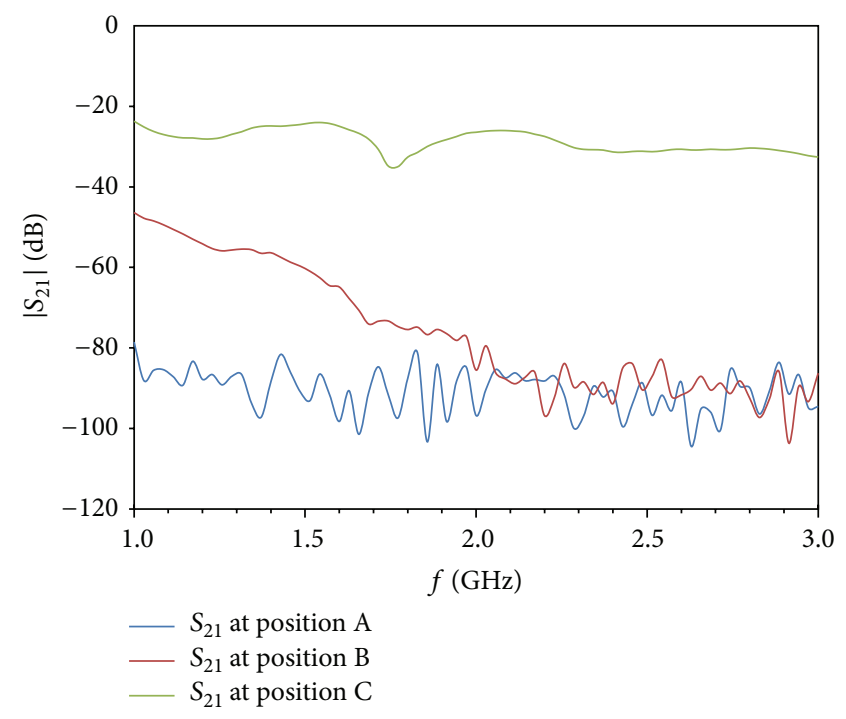

FIGURE 8: $\left|S_{21}\right|$ measurement when the transmitting antenna is placed at positions $\mathrm{A}, \mathrm{B}$, and $\mathrm{C}$.

position $\mathrm{B}$ this distance is $1 \mathrm{~m}$, whereas the $\mathrm{C}$ position is just over the PVC pipe where the victim is placed.

The measurements were performed in November, and the excavated soil was very wet, a worst-case scenario for breathing detection.

Figure 8 shows the $\left|S_{21}\right|$ between the receiving antenna placed on the victim's chest and the transmitting antenna at positions $\mathrm{A}, \mathrm{B}$, and $\mathrm{C}$, respectively.

It can be noticed that in position $\mathrm{A}$ the value is between -80 and $-100 \mathrm{~dB}$, highlighting a too high attenuation to detect the victim respiration; at position $\mathrm{B}$, from 1 to $2 \mathrm{GHz}$, sensitivity is higher, so the breathing measurement can be performed. The same measurements were also performed at position $\mathrm{C}$ where the attenuation is due to the PVC pipe only.

Figure 9 (blue line) reports the breathing frequency monitored at position $\mathrm{B}$. The fundamental respiration frequency is $0.2 \mathrm{~Hz}$ but higher harmonics can be observed at $0.4,0.6$, and $0.8 \mathrm{~Hz}$.

Since, for safety reasons, all persons who access the site must wear a high visibility jacket, an aluminum sheet was 


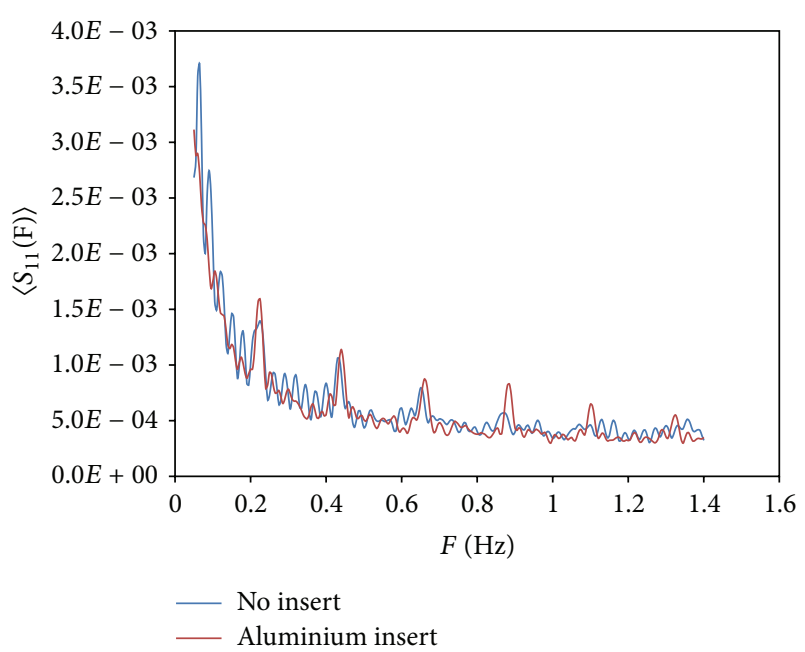

FIGURE 9: Breathing frequency detection at position B without (blue line) and with (red line) the insertion of an aluminum sheet in the high visibility jacket.

introduced in the jacket, improving the capability of the system to detect the thorax movements (red line).

\section{Discussion}

In this work an electromagnetic model to assess the design criteria for a rescue system has been presented; the model has been validated and finally the performances of a system, built up with laboratory equipment and designed according to the defined criteria, were tested to search a victim in a realistic scenario, as a road construction site. It is worth noting that during the study we realized that this system can be potentially used for other applications besides detecting the breathing activity of a victim. In fact the received signal can be processed in real time, and performing an Inverse Fourier Transform of the whatever column of (5), the subject position can be also retrieved. In case of multiple victims it is therefore possible to detect how many persons are buried under the landsleight and the rescue team would be able to assign the intervention priority depending on the vital signs of each victim.

The system could be also used in rescuing activities after other natural disasters as earthquake or avalanches, where the lossy medium is the rubble of the collapsed building and the snow, respectively. In this case further studies are required to analyze the signal propagation through a medium of random and inhomogeneous characteristics. In particular the antenna and the frequency range have to be finalized for the specific environment.

The capability of the system to sense the breathing activity could be also useful in many hospital applications; however, the patient must be monitored remotely without using sensors directly applied on the patient skin. Due to this characteristic and its possibility to monitor dressed patients or covered by blankets, the device could find its optimal applications in hospital departments such as burnt wards or the neonatal divisions.
The proposed system could also be used in Ambient Assist Living applications thanks to its capability to sense remotely human vital signs without compromising user privacy; the device seems to be particularly suitable to monitor domestic activity of elderly people because it requires neither interactions with the monitored subject nor training sessions.

\section{Conclusions}

In this work the usefulness of an EM model to assess the design constraints of a rescue system able to detect the breathing activities of buried victims was shown.

The model is analytical and simple, but effective and flexible. It was developed to simulate the behavior of an electromagnetic wave impinging on a human body buried under a lossy medium. The model allows us to determine some system design characteristics: (a) the optimal frequency range is $1-2 \mathrm{GHz}$, a tradeoff between the need for frequencies as high as possible to maximize the phase variations due to a respiratory act and the effect of soil attenuation; (b) a frequency sweep analysis in the optimal range is more suitable than a CW based system to avoid frequencies with a very low sensitiveness because of multiple reflections of the surrounding environment. On the other hand a UWB pulse could be characterized by a spectrum having most of its components strongly attenuated in case of lossy media; (c) the antenna would be well matched to reduce the $S_{11}$ term not correlated to the victim breath (4). This condition should be verified considering the antenna imbedded into the medium where the victim is buried.

\section{Conflict of Interests}

The authors declare that there is no conflict of interests regarding the publication of this paper.

\section{Acknowledgments}

The authors wish to thank STRABAG and in particular Eng. Cesare Avignone and Eng. Arturo Ercole for making available their site areas to test the system in a real environment.

\section{References}

[1] C. Li, V. M. Lubecke, O. Boric-Lubecke, and J. Lin, "A review on recent advances in doppler radar sensors for noncontact healthcare monitoring," IEEE Transactions on Microwave Theory and Techniques, vol. 61, no. 5, pp. 2046-2060, 2013.

[2] Y.-J. Chang, C.-H. Chen, L.-F. Lin, R.-P. Han, W.-T. Huang, and G.-C. Lee, "Wireless sensor networks for vital signs monitoring: application in a nursing home," International Journal of Distributed Sensor Networks, vol. 2012, Article ID 685107, 12 pages, 2012.

[3] C. I. Franks, B. H. Brown, and D. M. Johnston, "Contactless respiration monitoring of infants," Medical and Biological Engineering, vol. 14, no. 3, pp. 306-312, 1976.

[4] J. Y. Lee and J. C. Lin, "A microprocessor-based non-invasive arterial pulse wave analyzer," IEEE Transactions on Biomedical Engineering, vol. 32, no. 6, pp. 451-455, 1985. 
[5] K.-M. Chen, D. Misra, H. Wang, H.-R. Chuang, and E. Postow, "An X-band microwave life-detection system," IEEE Transactions on Biomedical Engineering, vol. 33, no. 7, pp. 697-701, 1986.

[6] B. Lohman, O. Boric-Lubecke, P. W. Ong, and M. M. Sondhi, "A digital signal processor for Doppler radar sensing of vital signs," IEEE Engineering in Medicine and Biology Magazine, vol. 21, no. 5, pp. 161-164, 2002.

[7] O. Boric-Lubecke, V. M. Lubecke, A. Host-Madsen, D. Samardzija, and K. Cheung, "Doppler radar sensing of multiple subjects in single and multiple antenna systems," in Proceedings of the 7th International Conference on Telecommunications in Moderm Satellite, Cable and Broadcasting Services, vol. 1, pp. 711, September 2005.

[8] Q. Zhou, J. Liu, H. A. Madsen, O. Boric-Lubecke, and V. Lubecke, "Detection of multiple heartbeats using Doppler Radar," in Proceedings of the IEEE International Conference on Acoustics, Speech and Signal Processing (ICASSP '06), vol. 1, pp. 1160-1163, Toulouse, France, May 2006.

[9] C. Li and J. Lin, "Random body movement cancellation in doppler radar vital sign detection," IEEE Transactions on Microwave Theory and Techniques, vol. 56, no. 12, pp. 3143-3152, 2008.

[10] M. D'Urso, G. Leone, and F. Soldovieri, "A simple strategy for life signs detection via an $\mathrm{x}$-band experimental set-up," Progress in Electromagnetics Research C, vol. 9, pp. 119-129, 2009.

[11] C. Li, Y. Xiao, and J. Lin, "Experiment and spectral analysis of a low-power Ka-band heartbeat detector measuring from four sides of a human body," IEEE Transactions on Microwave Theory and Techniques, vol. 54, no. 12, pp. 4464-4471, 2006.

[12] B.-K. Park, O. Boric-Lubecke, and V. M. Lubecke, "Arctangent demodulation with DC offset compensation in quadrature Doppler radar receiver systems," IEEE Transactions on Microwave Theory and Techniques, vol. 55, no. 5, pp. 1073-1078, 2007.

[13] F.-K. Wang, C.-J. Li, C.-H. Hsiao et al., "A novel vital-sign sensor based on a self-injection-locked oscillator," IEEE Transactions on Microwave Theory and Techniques, vol. 58, no. 12, pp. 41124120, 2010.

[14] A. G. Yarovoy, L. P. Ligthart, J. Matuzas, and B. Levitas, "UWB radar for human being detection," IEEE Aerospace \& Electronic Systems Magazine, vol. 21, no. 11, pp. 22-26, 2006.

[15] A. Nezirović, A. G. Yarovoy, and L. P. Ligthart, "Signal processing for improved detection of trapped victims using UWB radar," IEEE Transactions on Geoscience and Remote Sensing, vol. 48, no. 4, pp. 2005-2014, 2010.

[16] J. Li, L. Liu, Z. Zeng, and F. Liu, "Advanced signal processing for vital sign extraction with applications in UWB radar detection of trapped victims in complex environments," IEEE Journal of Selected Topics in Applied Earth Observations and Remote Sensing, vol. 7, no. 3, pp. 783-791, 2014.

[17] P. Russo, V. M. Primiani, A. De Leo, and G. Cerri, "Radiated susceptibility of breath monitoring system based on UWB pulses in spacecraft modules," in Proceedings of the International Symposium on Electromagnetic Compatibility (EMC EUROPE '12), vol. 1 of 4, p. 1, Rome, Italy, September 2012.

[18] P. Russo, V. M. Primiani, A. De Leo, and G. Cerri, "Radiated emission of breath monitoring system based on UWB pulses in spacecraft modules," in Proceedings of the ESA Workshop on Aerospace (EMC '12), vol. 1, pp. 1-6, Venice, Italy, May 2012.

[19] I. Arai, "Survivor search radar system for persons trapped under earthquake rubble," in Proceedings of the Asia-Pacific Microwave Conference (APMC '01), vol. 2, pp. 663-668, Taipei, Taiwan, December 2001.
[20] C. Labarthe, J. Mutzig, B. Jecko et al., "An Ultra-Wideband radar concept for the detection of buried victims beneath building rubble," in Proceedings of the International Radar ConferenceSurveillance for a Safer World (RADAR '09), pp. 1-6, IEEE, Bordeaux, France, October 2009.

[21] J. Li, Z. Zeng, J. Sun, and F. Liu, "Through-wall detection of human being's movement by UWB radar," IEEE Geoscience and Remote Sensing Letters, vol. 9, no. 6, pp. 1079-1083, 2012.

[22] G. Grazzini, M. Pieraccini, F. Parrini et al., "An ultra-wideband high-dynamic range GPR for detecting buried people after collapse of buildings," in Proceedings of the 13th Internarional Conference on Ground Penetrating Radar (GPR '10), pp. 1-6, June 2010.

[23] A. de Leo, V. M. Primiani, P. Russo, D. Shahu, V. di Mattia, and G. Cerri, "Breath detection of humans buried in a homogeneous lossy medium: a simplified analytical model," in Proceedings of the International Symposium on Electromagnetic Compatibility (EMC EUROPE '12), pp. 1-6, 2012.

[24] A. De Leo, V. Petrini, P. Russo, V. Mariani Primiani, L. Scalise, and G. Cerri, "A breathing detection medical device for rescue of buried people," in Proceedings of the International Symposium on Electromagnetic Compatibility (EMC Europe '14), pp. 901905, 2014.

[25] W. R. Scott Jr. and G. S. Smith, "Measured electrical constitutive parameters of soil as functions of frequency and moisture content," IEEE Transactions on Geoscience and Remote Sensing, vol. 30, no. 3, pp. 621-623, 1992. 

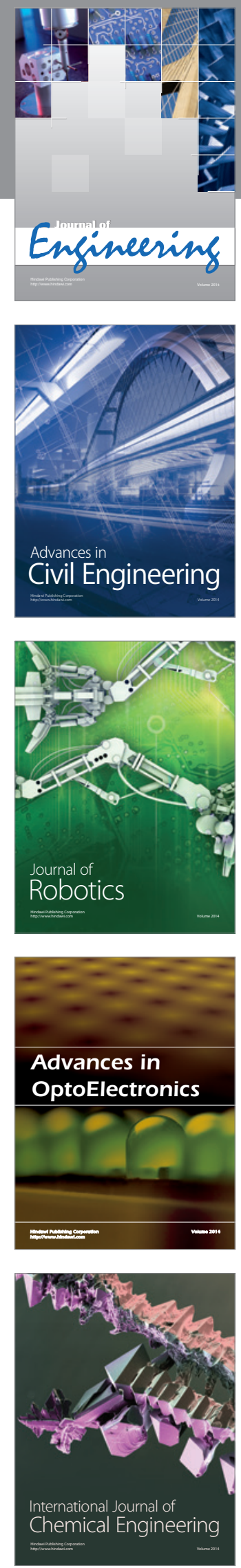

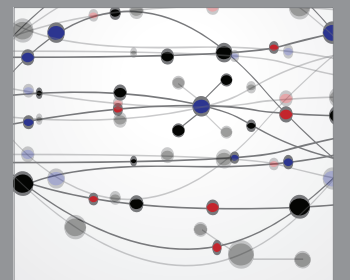

The Scientific World Journal
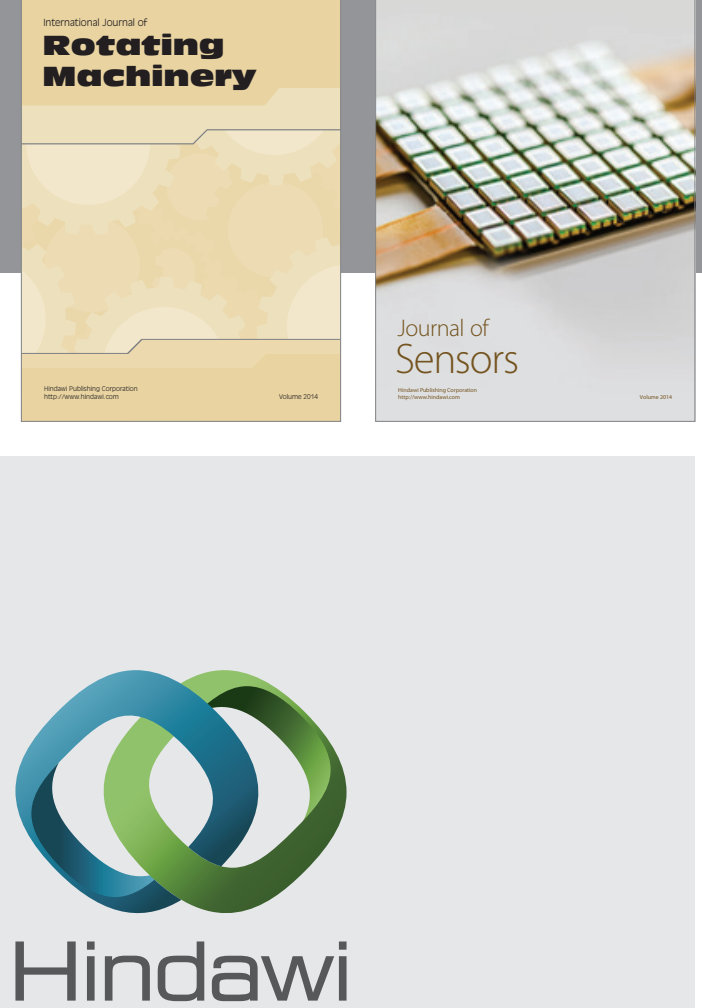

Submit your manuscripts at http://www.hindawi.com
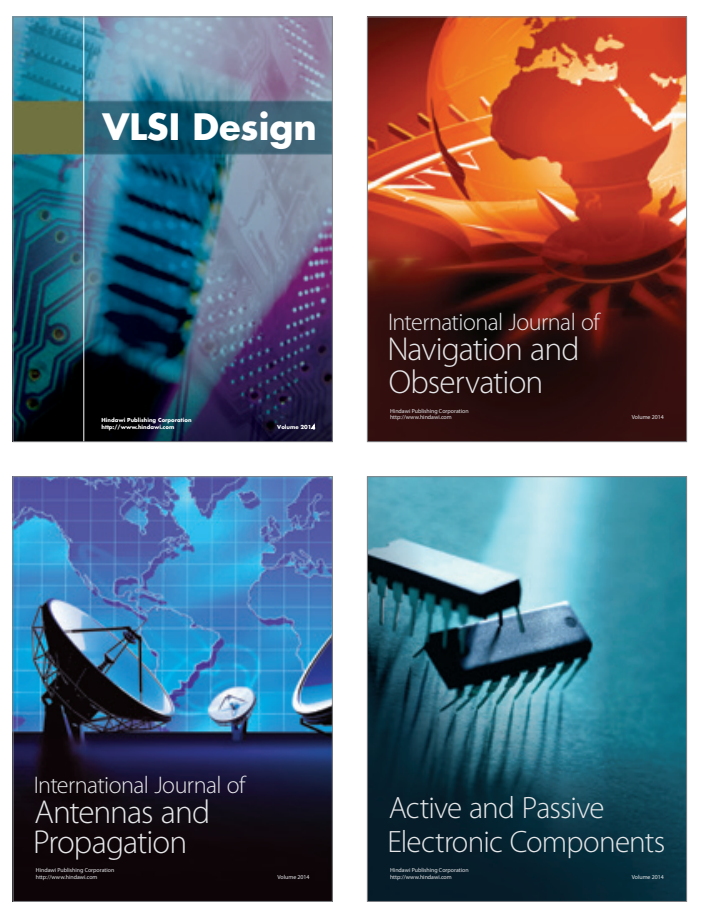
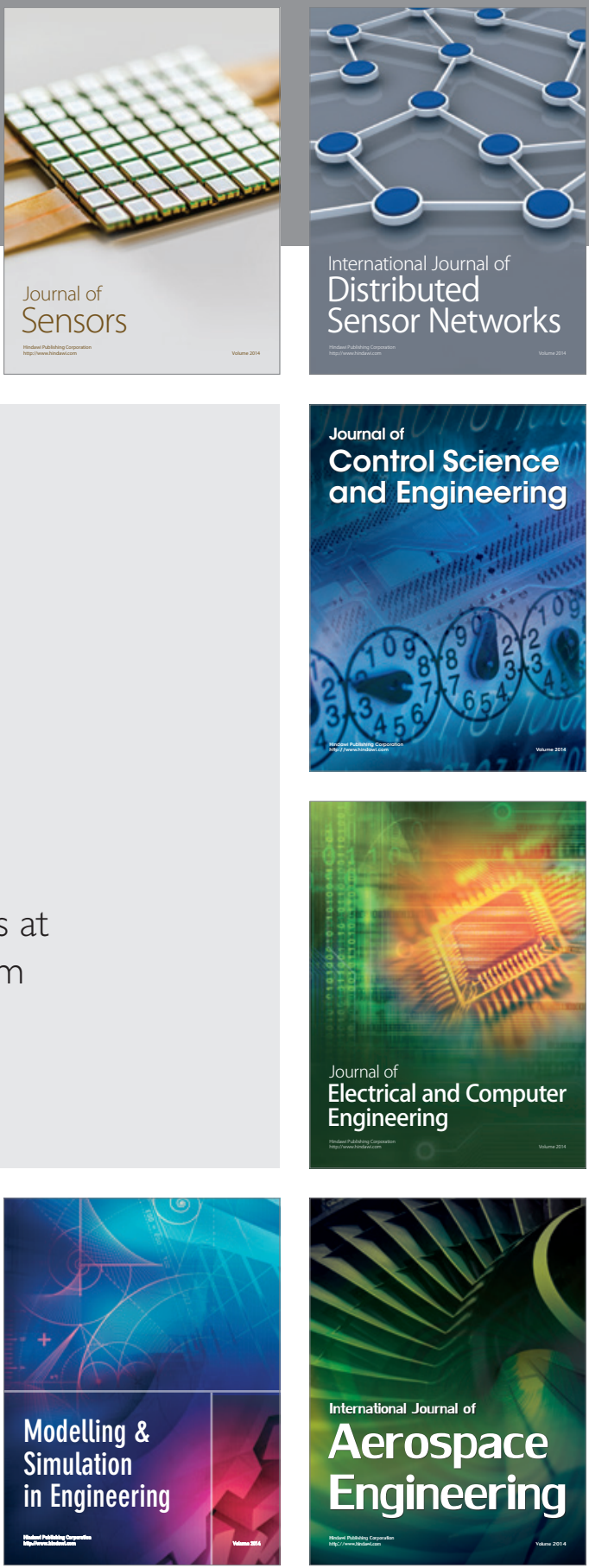

Journal of

Control Science

and Engineering
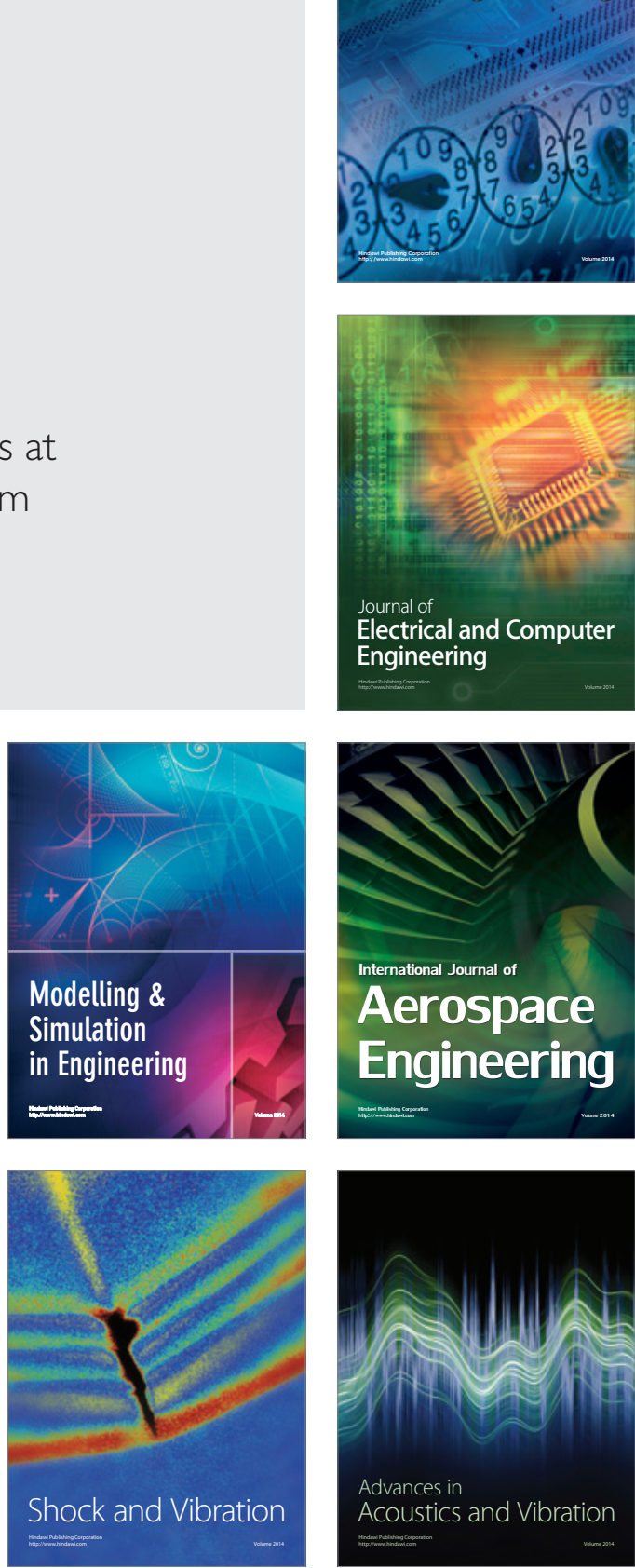